\title{
In vivo Evaluation of FimH Antagonists - A Novel Class of Antimicrobials for the Treatment of Urinary Tract Infection
}

\author{
Daniela Abgottspon ${ }^{\S *}$ and Beat Ernst \\ §SCS-Metrohm Foundation Award for best oral presentation
}

\begin{abstract}
The discovery of antimicrobials as $\beta$-lactam antibiotics or aminoglycosides revolutionized the treatment of infectious diseases. However, the extensive use rapidly created the problem of resistant pathogens, which are increasingly difficult to treat. FimH antagonists are a new class of antimicrobials, which target the bacterial adhesion to urothelial cells, a crucial first step in the establishment of urinary tract infections. Because of their different mode of action, FimH antagonists neither kill nor inhibit the growth of bacteria, they should have a reduced potential to generate resistant strains. This mini-review outlines the main problems associated with increasing development of antimicrobial resistance. Furthermore, it summarizes the currently available in vivo studies in mice for the treatment of urinary tract infections conducted with FimH antagonists.
\end{abstract}

Keywords: FimH antagonists · $\alpha$-D-Mannopyranoside $\cdot$ Type 1 pili $\cdot$ Urinary tract infection (UTI) · Uropathogenic Escherichia coli (UPEC)

\section{Antibiotics and Resistance - The Microbiological Twins}

The discovery of $\beta$-lactame antibiotics in the 1930s as well as the introduction of hygienic standards in hospitals (hand washing, use of disinfectants) was an incisive step in the development of public healthcare. Humans gained control over a $10^{7}$ times smaller organism and the mortality from bacterial infections rapidly diminished and concomitantly also the interest to develop new antimicrobials. However, shortly after it was assumed that bacterial infections were under control, the first emergence of resistant strains evolved. The ability of our tiny cohabitants to adapt to new living conditions was severely underrated and only insufficient efforts were undertaken to contain the emerging bacterial threat with new antibiotics. ${ }^{[1]}$ Therefore,

\footnotetext{
${ }^{\star}$ Correspondence: D. Abgottspon University of Basel Institute of Molecular Pharmacy Klingelbergstrasse 50 $\mathrm{CH}-4056$ Basel

since the 1990s, infectious diseases have non-surprisingly developed to become one of the top five causes of death in high-income countries. ${ }^{[2]}$

The majority of the classes of antimicrobials still used today have their origin in the 1940s to 1970s (Fig. 1). ${ }^{[3]}$ Although numerous chemically modified derivatives entered the market in recent years, real innovation only occurred in the last 10 years when new classes of antibiotics were approved for clinical use (oxazolidinones, lipopeptides and mutilins). ${ }^{[4,11]}$ The diminished interest to develop new antibiotics and the rapidly increasing emergence of resistance led to the highly prevalent problem that for an increasing number of multidrug-resistant (MDR) and pandrugresistant (PDR) bacteria no effective therapeutic treatment is available. ${ }^{[4]}$ An essential cause for the emergence of antimicrobial resistance is the extensive use of antibiotics, leading to treatment failure, a reduced range of therapeutic options for consecu- tive bacterial infections and ultimately a serious threat for the patient. ${ }^{[12,13]}$

The most common bacterial infectious diseases are respiratory tract infections and urinary tract infections (UTIs). Although UTI is not a life-threatening disease, all symptomatic infections should be treated with antibiotics to prevent potential devastating complications, like pyelonephritis and urosepsis. Thus, the availability of a novel class of antimicrobials based on an alternative mode of action would have a huge impact on the treatment of UTI, being a substantial contribution to public health. ${ }^{[14]}$

\section{Adhesion and Infection - The Importance of the FimH Lectin for UTIs}

The crucial step for the majority of infectious diseases is the initial contact (adhesion) of the infecting microorgan-

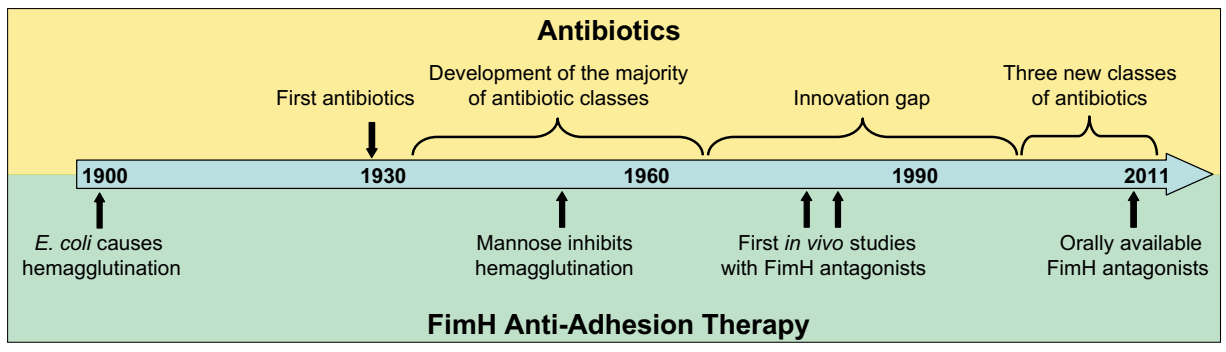

Fig. 1. Timeline of the major steps of the development of antibiotics (top $\left.{ }^{[4]}\right)$ and the FimH antiadhesion therapy for the treatment of UTI (bottom ${ }^{[5-10]}$ ). 
ism with the target cells, enabling bacteria to avoid the natural clearing mechanisms and the immune system and thereby ensure survival in the host environment. ${ }^{[14]}$ UTI is predominantly caused by urophathogenic Escherichia coli (UPEC) and strongly depends on the specific adhesion of the pathogen to carbohydrate-containing ligands on the host endothelium. ${ }^{[15]}$ The ability of UPEC to adhere to and to ascend within the urinary tract is mediated via filamentous multi-subunit membrane proteins, so-called type 1 pili and P-pili. ${ }^{[16]}$ Whereas P-pili account for the infection of the kidneys (pyelonephritis), type 1 pili are responsible for the initial colonization of the bladder (cystitis). The four subunits FimA, FimF, FimG and FimH compose a type 1 pilus, with FimH located at the tip. ${ }^{[17]}$ As a part of the FimH subunit, a carbohydrate-recognition domain (CRD) is responsible for bacterial attachment to oligomannosides of the glycoprotein uroplakin Ia (UPIa) located on the urinary bladder mucosa. ${ }^{[18]}$ This initial step prevents the rapid clearance of $E$. coli from the urinary tract by the bulk flow of urine and at the same time, initiates the infection process (Fig. 2A). ${ }^{[19]}$

\section{Virulence and FimH - A Target for Anti-Adhesion Therapy}

Previous studies clearly demonstrated that type 1 pili deficient UPEC strains are not able to initiate an infection in mice. [21] Furthermore, the immunization of mice with antibodies directed against FimH, protected animals from bladder-colonization with UPEC. ${ }^{[22]}$ These findings strongly suggest that the inhibition of the initial host-pathogen interaction with FimH antagonists as a suitable approach to prevent and treat UTIs.

The host defense system exerts a FimH related mechanism to protect the sterile urinary tract from invading UPECs. The most abundant protein in the urine is the Tamm-Horsfall protein (THP; also called uromodulin). It is a high-mannosylated glycoprotein and is exclusively produced in the kidneys. It is able to neutralize UPEC by binding to their type 1 pili and it thereby prevents the adhesion to urothelial cells. ${ }^{[23]}$ Interestingly, it is highly conserved during evolution and THP-deficient mice are more susceptible to UTI. ${ }^{[24]}$ Once the bacteria are trapped by THP, the THP/bacteria cluster is eliminated by the urine flow from the urinary tract. FimH antagonists would support the host defense mechanism to clear excessively invading bacteria. In addition, type 1 pili exhibit several important target-qualities for the development of an anti-adhesion therapy: (i) they are the most prevalent fimbriae encoded by UPEC, (ii)

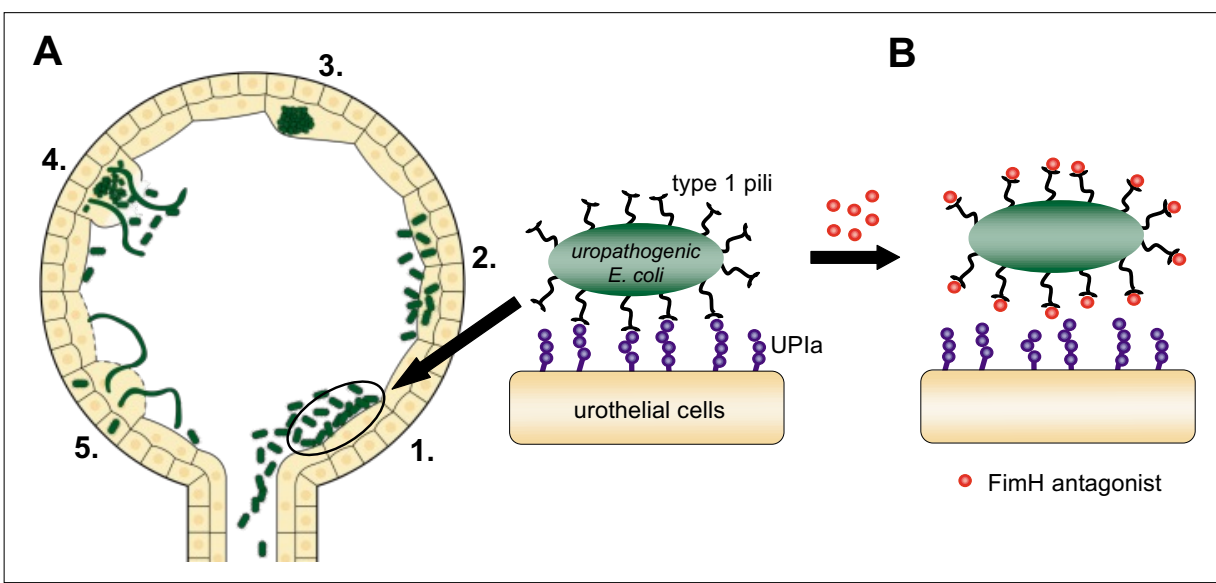

Fig. 2. A) Urinary tract infection cycle. 1. The first and most important step in the development of UTI is the type 1 pili dependent adhesion of UPEC to uroplakin la (UPla) on urothelial cells. 2. Adhesion triggers the invasion of the bacteria into the superficial bladder cells, lining the urinary tract. Once inside the cells, bacteria start to replicate and form 3. intracellular biofilms, where they are well protected from the host defense mechanisms and antibiotic treatment. 4. Later on, cells start to exfoliate and bacteria exit the cells in filamentous structures and 5. disperse in the environment ready to infect new cells (infection cycle adapted from ref. [20]). B) In the presence of FimH antagonists, UPECs are not able to adhere to urothelial cells and are therefore washed out with the flow of urine. As a result, the infection cycle cannot be established. they are highly conserved during evolution and (iii) they mediate the adhesion, which is the first and most important step for the establishment of the infection cycle (Fig. 2).

\section{UTI Mouse Model and Treatment - The Therapeutic Potential of FimH Antagonists}

\subsection{Initial in vivo Studies}

In the 1970s, Sharon and coworkers pioneered the discovery of anti-adhesion molecules targeting FimH. They reported on the in vitro inhibitory potential of methyl $\alpha$-D-mannopyranoside (1) ${ }^{[25]}$ (Fig. 3) and $p$-nitrophenyl $\alpha$-D-mannopyranoside, ${ }^{[26]}$ and investigated (in vitro and in vivo) the FimH lectin as a potential target for an antiadhesion therapy. In the first in vivo proof of concept study, FimH antagonist $\mathbf{1}$ was pre-incubated with type 1 pili expressing UPEC strains and the mixture was instilled transurethrally into the bladder of mice. ${ }^{[7]}$ Urine samples were analyzed every two to three days for bacterial counts, resulting in a significant reduction of bacteriuric mice after 5-16 days. In a second experiment, an additional dose of $\mathbf{1}$ was injected intra- peritoneally two and three days after the infection with the bacteria/antagonist mixture, leading to no significant supplemental benefit. As mentioned by Sharon and coworkers, ${ }^{[7]}$ a major drawback of the UTI mouse model is the spontaneous clearance of bacteria from the urinary tract 14 days after infection (up to $45 \%$ ). Nevertheless, when the sampling of urine was conducted for up to 16 days, a significant reduction of bacteriuric mice treated with the FimH antagonist compared to the control group was observed. Based on this promising in vivo experiment, research directed to the identification of improved FimH antagonists ${ }^{[27]}$ as well as the elucidation of the underlying mechanisms of this host-pathogen interaction (e.g. type 1 and $\mathrm{P}$-pili, ${ }^{[28]}$ phase variation of type 1 pili, ${ }^{[29]}$ immune response, ${ }^{[30]}$ etc.) was initiated.

In the 1980s, Svanborg Edén et al. ${ }^{[8]}$ repeated the in vivo study ${ }^{[7]}$ with methyl $\alpha$-D-mannopyranoside (1) by instilling a higher concentration of bacteria/antagonist mixture into the bladder of mice. 2 and $16 \mathrm{~h}$ after the onset of infection, the animals were sacrificed to analyze their bladder and kidneys for bacterial counts. Surprisingly, they reported no reduction of bacterial load, although they used a higher

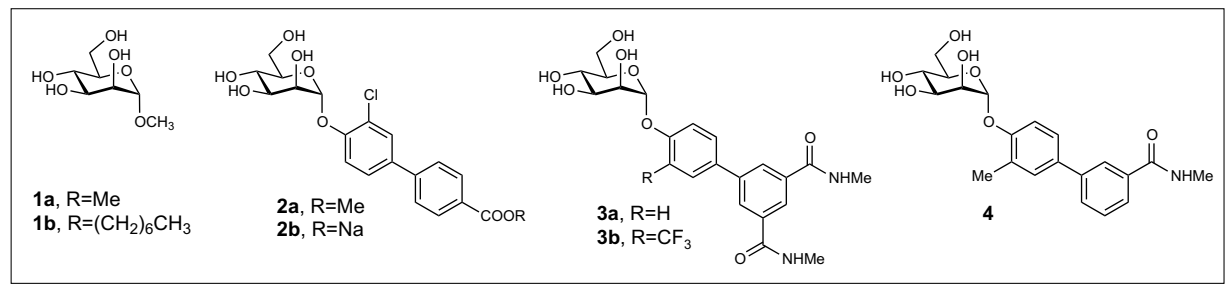

Fig. 3. $\alpha$-D-Mannopyranosides tested in the UTI mouse model. Methyl and n-heptyl $\alpha$-Dmannopyranosides $\mathbf{1 a}$ and $\mathbf{1 b},{ }^{[7-9]}$ biphenyl $\alpha$-D-mannopyranoside $\mathbf{2 a}$ and $\mathbf{2} \mathbf{b},{ }^{[9]}$ diamidobiphenyl $\alpha$-D-mannopyranoside $\mathbf{3 a}$ and $\mathbf{3} \mathbf{b}^{[10]}$ and monoamidobiphenyl $\alpha$-D-mannopyranoside $4 .{ }^{[10]}$ 
inoculation concentration of $\mathbf{1}$ compared to the in vivo experiment of Sharon and coworkers $^{[7]}(5 \mathrm{mg} / \mathrm{ml}$ vs. $0.1 \mathrm{mg} / \mathrm{ml})$. The different outcome of the two in vivo experiments could be related to the applied bacterial strains. Both groups used clinical UPEC isolates for their in vivo studies, however, Svanborg Edén et al. ${ }^{[8]}$ subjected their strain to chemical mutagenesis by treatment with $\mathrm{N}$-methyl-N-nitro-Nnitrosoguanidine, leading to random mutations. ${ }^{[31]}$ Modified binding properties could therefore explain the different outcome of the two in vivo experiments. Furthermore, methyl $\alpha$-D-mannopyranoside (1) is a low affinity antagonist ${ }^{[32]}$ and would probably require much higher concentrations for the high level of inoculation (up to $10^{9}$ UPECs per mouse, ${ }^{[8]}$ compared to $10^{6}$ UPECs by Sharon and coworkers $\left.{ }^{[7]}\right)$.

After these initial in vivo studies investigating the therapeutic potential of FimH antagonists, ${ }^{[7,8]}$ no further in vivo studies were published for almost three decades (Fig. 1). At the same time, however, the UTI mouse model was extensively used for in depth investigations of the basic mechanisms of bacteria associated UTIs. ${ }^{[16]}$ Simultaneously, the development of more potent FimH antagonists followed two major directions. First, multivalent FimH antagonists were investigated ${ }^{[33]}$ and second, based on structural information obtained from the crystal structure of FimH co-crystallized with antagonists, ${ }^{[34]}$ monovalent, high-affinity antagonists were designed ${ }^{27]}$ (the design, synthesis and in vitro evaluation of mono- and oligovalent mannosides is reviewed in detail by Hartmann and Lindhorst, ${ }^{[27]}$ and Ernst and Magnani ${ }^{[35]}$ ).

\subsection{Orally Available FimH \\ Antagonists}

Recently, Klein et al. ${ }^{[9]}$ published for the first time in vitro and in vivo pharmacokinetic (PK) data of monovalend FimH antagonists. For oral availability, an optimal balance between solubility, permeability and lipophilicity is required. In addition, to ensure the availability of the antagonist at the target organ (bladder), a prodrug approach was applied. The ester $\mathbf{2 a}$ is orally absorbed and hydrolyzed to the renally excretable acid $\mathbf{2 b}$. When applied to the UTI mouse model (single dose of 50 $\mathrm{mg} / \mathrm{kg}$, p.o.) ten minutes prior to infection, a substantial reduction of colony forming units (up to $-4 \log _{10} \mathrm{CFU}$ ) in the bladder could be detected.

In a recent study by Cusumano et al. ${ }^{[10]}$ the FimH antagonists were evaluated for their potential to treat as well as to prevent UTI in mice. For this purpose, animals with a two-week chronic infection were treated with 3a (single dose $100 \mathrm{mg} / \mathrm{kg}$ p.o.) or $\mathbf{3 b}$ and 4 (single dose of $50 \mathrm{mg} / \mathrm{kg}$ p.o.), resulting in an $3 \log _{10}$ CFU reduction of blad- der counts for $\mathbf{3 a}$ and up to $-4 \log _{10}$ CFU for $\mathbf{3 b}$ and $\mathbf{4}$ six hours after treatment. In comparison, the antibiotic trimethoprimsulfamethoxazole (TMP-SMZ, applied in the drinking water) only reduced bacterial counts by $1.5 \log _{10}$ CFU. Furthermore, antagonists applied together with antibiotics were reported to have a synergistic effect on the treatment outcome. In a 24 hours multiple dosage study (3 times $50 \mathrm{mg} / \mathrm{kg}$ of 4, every eight hours) for the treatment of chronic infections, bacterial counts in the bladder were reduced by $-4.5 \log _{10}$ CFU. Furthermore, to prevent infection, a single dose of $3 \mathbf{a}(50 \mathrm{mg} / \mathrm{kg})$ was applied $30 \mathrm{~min}$ utes prior to infection, resulting in a -1.5 $\log _{10}$ CFU reduction of bladder counts, $6 \mathrm{~h}$ after infection. Additionally, these authors reported that treatment with FimH antagonists prevented invasion of UPECs into the bladder cells leading to a reduction of biofilm formation.

In summary, both studies confirmed the promising potential of orally applied FimH antagonists for both prevention and treatment of UTIs in vivo. In addition, FimH antagonists proved to be equally effective as antibiotics. Whereas in the initial treatment experiments ${ }^{[7,8]}$ bacteria/antagonist mixtures were directly applied into the bladder, the latter studies ${ }^{[9,10]}$ used a therapeutically more relevant protocol by applying the FimH antagonists either p.o. or i.v., which closer simulates conditions used for later patient application. Furthermore, the higher potency of the newly developed FimH antagonists, further contributed to the positive therapeutic outcome in the UTI mouse model. However, the orally applied antagonists (2a, 3a, 3b \& 4) still exhibit unfavorable PK properties (e.g. low solubility, short exposure in plasma and urine), which both research teams aim to improve with further investigations.

\section{Summary and Outlook}

Although the development of resistant bacteria started shortly after the introduction of the first antimicrobial drugs, ${ }^{[13]}$ the treatment paradigm only recently shifted from killing the pathogen to inhibiting its adhesion to the host cells. The antiadhesion therapy is focusing on the development of a new class of antimicrobials exhibiting less selection pressure and therefore a reduced potential for the emergence of resistance. In addition, a reduction of the antibiotic associated side effects (e.g. the disruption of the commensal microbiota) is expected. A recent very promising example is the inhibition of the type 1 pili dependent adhesion of uropathogenic Escherichia coli (UPEC) to bladder cells for the treatment of urinary tract infections (UTIs). The in vivo studies reported to this day clearly indicate the potential of orally available FimH antagonists for the prevention and treatment of UTIs, with a higher therapeutic effect compared to treatment with antibiotics.

Received: January 23, 2012

[1] I. Ofek, D. L. Hasty, R. J. Doyle, 'Bacterial Adhesion to Animal Cells and Tissues', ASM Press, 2003.

[2] WHO statistics, 'The top 10 causes of death', Fact sheet $\mathrm{N}^{\circ} 310$, updated June 2011.

[3] K. J. Simmons, I. Chopra, C. W. G. Fishwick, Nat. Rev. Microbiol. 2010, 8, 501.

[4] M. A. Fischbach, C. T. Walsh, Science 2009, 325,1089

[5] G. Guyot, Zentbl. Bacteriol. Abt. I Orig. 1908, 47, 640 .

[6] W. A. Collier, J. C. deMiranda, Antonie Leeuwenhoek 1955, 21, 133.

[7] M. Aronson, O. Medalia, L. Schori, D. Mirelman, N. Sharon, I. Ofek, J. Infect. Dis. 1979, 139, 329

[8] C. Svanborg Edén, R. Freter, L. Hagberg, R. Hull, S. Hull, H. Leffler, G. Schoolnik, Nature 1982, 298, 560

[9] T. Klein, D. Abgottspon, M. Wittwer, S Rabbani, J. Herold, X. Jiang, S. Kleeb, C. Lüthi, M. Scharenberg, J. Bezençon, E. Gubler, L. Pang, M. Smiesko, B. Cutting, O. Schwardt, B. Ernst, J. Med. Chem. 2010, 53, 8627.

[10] C. K. Cusumano, J. S. Pinkner, Z. Han, S. E. Greene, B. A. Ford, J. R. Crowley, J. P. Henderson, J. W. Janetka, S. J. Hultgren, Sci. Transl. Med. 2011, 3, 109.

[11] B. Hamad, Nat. Rev. Drug Discov. 2010, 9, 675.

[12] H. Kresse, M. J. Belsey, H. Rovini, Nat. Rev. Drug Discov. 2007, 6, 19

[13] H. K. Allen, J. Donato, H. H. Wang, K. A. Cloud-Hansen, J. Davies, L. Handelsmann, Nat. Rev. Microbiol. 2010, 8, 251.

[14] N. Sharon, Biochim. Biophysi. Acta 2006, 1760 , 527.

[15] J. Berglund, S. D. Knight, Adv. Exp. Med. Biol. 2003, 535, 33.

[16] M. A. Mulvey, Cell Microbiol. 2002, 4, 257.

[17] G. Capitani, O. Eidam, R. Glockshuber, M. G. Grütter, Microbes Infect. 2006, 8, 2284.

[18] M. A. Mulvey, Cell Microbiol. 2002, 4, 257.

[19] T. J. Wiles, R. R. Kulesus, M. A. Mulvey, Exp. Mol. Pathol. 2008, 85, 11 .

[20] M. A. Mulvey, J. D. Schilling, J. J. Martinez, S. J. Hultgren, PNAS 2001, 97, 8829.

[21] H. Connell, W. Agace, P. Klemm, M. Schembri, S. Mårild, C. Svanborg, PNAS 1996, 93, 9827.

[22] S. Langermann, S. Palaszynski, M. Barnhart, G. Auguste, J. S. Pinkner, J. Burlein, P. Barren, S. Koenig, S. Leath, C. H. Jones, S. J. Hultgren, Science 1997, 276, 607.

[23] J. Pak, Y. Pu, Z. Zhang, D. L. Hasty, X. Wu, J. Biol. Chem. 2001, 276, 9924.

[24] J. M. Bates, H. M. Raffi, K. Prasadan, R. Mascarenhas, Z. Laszik, N. Maeda, S. J. Hultgren, S. Kumar, Kidney Int. 2004, 65, 791.

[25] I. Ofek, D. Mirelman, N. Sharon, Nature 1977, 265,623 .

[26] Y. Eshdat, I. Ofek, Y. Yashouv-Gan, N. Sharon, D. Mirelman, Biochem. Biophys. Res. Commun. 1978, 85,1551

[27] M. Hartmann, T. K. Lindhorst, Eur. J. Org. Chem. 2011, 3583.

[28] L. Hagberg, R. Hull, S. Hull, S. Falkow, R. Freter, C. Svanborg Edén, Infect. Immun. 1983, 40, 265.

[29] a) B. I. Eisenstein, Science 1981, 214, 337; b) S. J. Hultgren, T. N. Porter, A. J. Schaeffer, J. L. Duncan, Infect. Immun. 1985, 50, 370. 
[30] P. de Man, C. van Kooten, L. Aarden, I. Engberg, H. Linder, C. Svanborg Edén, Infect. Immun. 1989, 57, 3383.

[31] R. Arshad, S. Farooq, S. S. Ali, Ann. Microbiol. 2010, 60, 645 .

[32] a) S. Rabbani, X. Jiang, O. Schwardt, B. Ernst, Anal. Biochem. 2010, 407, 188; b) D. Abgottspon, G. Rölli, L. Hosch, A. Steinhuber X. Jiang, O. Schwardt, B. Cutting, M. Smiesko, U. Jenal, B. Ernst, A. Trampuz, J. Microbiol. Methods 2010, 82, 249; c) M. Scharenberg, D. Abgottspon, E. Ciceck, X. Jiang, O. Schwardt,
S. Rabbani, B. Ernst, Assay Drug Dev. Technol. 2011, 9, 455 .

[33] a) R. J. Pieters, Org. Biomol. Chem. 2009, 7, 2013. b) A. Imberty, Y. M. Chabre, R. Roy, Chem. Eur. J. 2008, 14, 7490.

[34] a) D. Choudhury, A. Thompson, V. Stojanoff, S. Langermann, J. Pinkner, S. J. Hultgren, S. D. Knight, Science 1999, 285, 1061; b) J. Bouckaert, J. Berglund, M. Schembri, E. D. Genst, L. Cools, M. Wuhrer, C. S. Hung, J. Pinkner, R. Slättergard, A. Zavialov, D. Choudhury, S. Langermann, S. J. Hultgren, L.
Wyns, P. Klemm, S. Oscarson, S. D. Knight, H. D. Greve, Mol. Microbiol. 2005, 55, 441; c) A. Wellens, C. Garofalo, H. Nguyen, N. Van Gerven, R. Slättergârd, J.-P. Hernalsteens, L. Wyns, S. Oscarson, H. De Greve, S. Hultgren, J. Bouckaert, PLos One 2008, 3, 4; d) Z. Han, J. S. Pinker, B. Ford, R. Obermann, W. Nolan, S. A. Wildman, D. Hobbs, T. Ellenberger, C. K. Cusumano, S. J. Hultgren, J. W. Janetka, J. Med. Chem. 2010, 53, 4779.

[35] B. Ernst, J. L. Magnani, Nat. Rev. Drug Discov. 2009, 8, 661 . 